\title{
Nanomedicine: promises and challenges for the future of public health
}

\author{
This article was published in the following Dove Press journal: \\ International Journal of Nanomedicine \\ 30 September 2010 \\ Number of times this article has been viewed
}

\section{Michelle Pautler \\ Sara Brenner \\ UAlbany College of Nanoscale Science and Engineering, Albany, NY, USA}

Correspondence: Sara Brenner, MD, MPH

UAlbany College of Nanoscale Science and Engineering, Nanobioscience

Constellation, 257 Fuller Road, NanoFab

East, Rm 4406, Albany, NY 12203, USA

$\mathrm{Tel}+\mathrm{I} 5189567224$

Fax + I 5184378687

Email sbrenner@uamail.albany.edu

\begin{abstract}
As the scope of nanotechnology applications in medicine evolves, it is important to simultaneously recognize and advance contributions germane to public health. A wide range of innovations in nanomedicine stand to impact nearly every medical specialty and unveil novel ways to improve the quality and extend the duration of life - these gains can be measured at both individual and population levels. For example, heart disease and cancer combined make up approximately half of all deaths in the United States per year, and already, advances in nanomedicine demonstrate great potential to reduce rates of morbidity and mortality due to these diseases. Meanwhile, public health applications of nanomedicine such as rapid and portable diagnostics and more effective vaccinations have the potential to revolutionize global health. Research driven by innovators across disciplines such as engineering, biology, medicine, and public health should collaborate in order to achieve maximal potential impact in health for individuals and populations. In turn, knowledge gaps regarding the potential health and safety implications of exposure to engineered nanomaterials must be continuously addressed and actively researched. Dynamic, proactive, and socially responsible research will drive nanomedicine as it plays an increasingly integral and transformative role in medicine and public health in the 21 st century.
\end{abstract}

Keywords: nanotechnology, medicine, epidemiology, policy, environmental health

\section{Introduction}

Applications of nanotechnology in the screening, diagnosis, and treatment of disease are collectively referred to as "nanomedicine" - an emerging field that has the potential to revolutionize individual and population-based health in the 21 st century. While clinical medicine targets health at the individual level, the mission of public health is to promote, protect, and preserve health for groups of people or populations. ${ }^{1}$ To maximize the benefits and minimize potential harms for the greatest number of people, it is essential to investigate and explore the potential applications and impacts of nanomedicine through the lens of public health.

The attractiveness of nanotechnology applications lies in the unique characteristics and phenomena that manifest due to their small size. The most widely accepted definition of scale for nanotechnology is $1-100 \mathrm{~nm}^{2}$ Engineering materials on this scale allows for novel medical therapies such as designing nanoparticle-based drugs that target cells with improved specificity, resulting in decreased side effects for patients. ${ }^{3}$ Other advances are being made in medical devices and instrumentation for use in surgical procedures that are less invasive, leading to shorter recovery times and decreased risk of postoperative infections or other complications. Such innovations will improve the quality of life, extend life expectancies, and could reduce the overall cost of healthcare. 
Research is currently underway on a global scale to develop nanotechnology applications in cardiology, neurology, and many other medical specialties. These applications range from nanoparticles in targeted drug delivery systems to carbon nanotubes for enhancing the diagnostic capacity of imaging modalities. ${ }^{4}$ Research driven by scientists with a keen eye on the needs of public health is essential in successfully advancing the field and developing medical technologies for practical use. At the same time, knowledge gaps regarding the fate and transport of engineered nanoparticles in biological systems need to be continuously addressed. ${ }^{5}$ In the United States, there are no specific regulations set forth by the United States Food and Drug Administration (FDA) for engineered nanomaterials in consumer products under their jurisdiction. However, there is growing concern about possible health and safety risks and the lack of information available to predict and mitigate potential health effects associated with exposure to engineered nanomaterials. Additional research needs to be conducted to adequately assess the life-cycle of engineered nanomaterials and how this will impact environmental and human health and safety. Meanwhile, communication with stakeholders about potential risks and benefits is critical to responsibly advance nanotechnology.

Implementation of nanomedicine into routine clinical practice will face many challenges from regulatory agencies, concerned public groups, insurance companies, and others for a variety of reasons. Collaboration between those with a vested interest in advancing nanomedicine should be encouraged to address these challenges at an early stage. Public health professionals should play an integral role in shaping future nanomedicine research as well as clinical and populationbased implementation. Their responsibilities will range from collecting and analyzing epidemiologic data on the effectiveness of nanoenabled drugs to advocating for increased funds to support research. With proper due diligence and collaboration, the fields of nanomedicine and public health will accelerate each other in a variety of ways to improve human health more efficiently than either could do individually.

The goal of this article is to educate stakeholders who include the general public, members of industry, government, and academia involved in advancing nanomedicine about public health concepts that should be proactively incorporated into the research, development, and implementation of this rapidly growing field.

\section{Public health}

Nanomedicine is poised to impact all aspects of public health. Public health is defined as "The science and art of preventing disease, prolonging life and promoting health and efficiency through organized community effort." This often includes community-wide or population-based interventions such as public sanitation, infectious disease control, and clinical preventive services including early screening and detection. In this way, public health works to promote the health of each person in a community through organized efforts that impact groups of people as well as individuals. ${ }^{6}$ The field of public health is broken down into the following five core disciplines according to the Association of Schools of Public Health: ${ }^{7}$

- Epidemiology

- Biostatistics

- Health policy management

- Social and community behavior

- Environmental health sciences.

Epidemiology is the study of determinants and distribution of disease. ${ }^{2}$ It articulates with biostatistics, which quantitatively analyzes the determinants and distribution of disease. Health policy and management uses information from disciplines in medicine and public health to create laws, regulations, and guidelines for the good of the public's health. Social and community behavior studies individual through organizational level impacts on health outcomes. ${ }^{7}$ Environmental health focuses on how the physical and social environment affects human health, as well as how humans are affecting their surrounding environment. ${ }^{8}$ The knowledge base achieved through these core disciplines provides a platform for public health professionals to analyze, understand, and predict the implications of nanomedicine on population health.

Technological advances in public health have shaped human history. For example, vaccines have been successful in eradicating and drastically reducing deadly infectious diseases across the globe. Side effects and health risks have also played a role in how vaccines have been developed and utilized. The hepatitis B vaccine is one example of how nanotechnology can be used to enhance current medical practice and make a significant impact on population health. Hepatitis B is an infectious disease that has affected about two billion people across the world. Currently there is a highly effective vaccine to prevent this disease; however, it must be administered over the course of three to four doses. ${ }^{9}$ Noncompliance with the dosing schedule is a significant problem, particularly in third world countries, rendering the vaccine less effective or completely ineffective. Ongoing research and development has led to breakthroughs that enable the vaccine to be administered in one dose with equal effectiveness. One group is specifically investigating the effectiveness of different PLGA microspheres to deliver 
the hepatitis B vaccine in one dose. ${ }^{10}$ This type of preventive care for hepatitis B makes it feasible to vaccinate a larger population against a significant public health threat - an infectious disease that leads to approximately 600,000 deaths a year. ${ }^{9}$

In terms of fighting chronic disease, nanomedicine has the potential to dramatically impact health within the United States as well as across the world. In the United States, cancer is the number two cause of mortality; it is estimated that in 2009, there were approximately 1.48 million cancer cases. ${ }^{11}$ Traditional cancer treatments such as chemotherapy, surgery, and radiation therapy are taxing on patients because these interventions are largely nonspecific; they damage both cancerous and healthy cells. ${ }^{12}$ The National Cancer Institute (NCI), recognizing the tremendous potential of nanotechnology to enhance the diagnosis and treatment of cancer, developed the Alliance for Nanotechnology in Cancer in 2004. ${ }^{13}$ This alliance fosters research and development of nanotechnology-based solutions ranging from diagnosis to treatment of cancer that are more efficient and have fewer side effects. The NCI provides support for many drugs and therapies harnessing nanotechnology in various phases of testing, all the way up through clinical trials. Currently, there are multiple nanoenabled drugs that have been approved by the FDA for the treatment of cancer, such as Abraxane ${ }^{\circledR}$, which is used to treat breast cancer, and Doxil ${ }^{\circledR}$ for ovarian cancer. ${ }^{14,15}$ Advances in regenerative medicine utilizing carbon nanotubes and nanofibers, nanopatterned extracellular matrices, and dendritic nanopolymers are a few examples of new technologies that are being developed to impact other major causes of morbidity and mortality in the United States, including cancer, Alzheimer's disease, and heart disease. ${ }^{16-19}$ Research efforts focused on these highly prevalent diseases demonstrate how nanomedicine can be used in a variety of ways to tackle large public health issues.

\section{Epidemiology and biostatistics}

Epidemiologic research is a powerful tool used to track health determinants and distribution of diseases in populations to manage health problems. ${ }^{2}$ Complementary to epidemiology is biostatistics, which is defined as the application of statistical methods to biological and medical problems. ${ }^{20}$ Research in these disciplines revealed correlations between exposures and health outcomes that have dramatically changed medicine and public health practices. Epidemiologic investigations range from environmental exposures that may result in a certain disease, or a new medical intervention that may reduce the incidence of a particular disease. In conjunction with biostatistics, epidemiologists will monitor the impact nanomedicine applications will have on particular health outcomes of interest as well as overall population health. This will include comprehensive post-market surveillance studies of nanoenabled drugs to monitor their safety as well as effectiveness. ${ }^{21}$

Monitoring advances in nanotechnology that have the potential to influence health determinants, goals, and outcomes through epidemiologic surveillance will allow researchers to quantify their impact on population health. It is important not only to investigate the question, "does the application of this technology improve health outcomes?" but also the critical question, "for whom does this technology improve health outcomes?" In the era of personalized medicine, we must demonstrate and validate the use of nanotechnology for individuals and groups based on pertinent physical, medical, and biological characteristics. Advances in personalized medicine through lab-on-a chip nanoarray technologies will assist in the quantification and understanding of how health outcomes are being affected by nanomedicine applications. ${ }^{22,23}$ The data and trend information generated by epidemiology will advance our understanding of the "big picture" impact of these technologies and also serve to reveal how they are advancing the health of people both nationally and internationally.

As nanomedicine applications become more mainstream in clinical care, information regarding how they are impacting health outcomes will help insurance companies make decisions based on cost-benefit analyses between new and traditional medical interventions. This could potentially drive down healthcare expenditures. In turn, this surveillance data will highlight areas of medical research that are in need of nanotechnology applications. Communication with federal agencies as to where these gaps persist will ensure that funding is being allocated to the areas with the greatest potential impact. Epidemiologic surveillance of where nanomedicine applications are primarily being used will demonstrate whether there is an equal distribution among various populations in different regions of the world. These considerations, as well as the public policy, societal, and ethical implications, are important elements in guiding future applications of nanotechnology in medicine and health.

\section{Health policy management}

The two major components regarding public health policy and management that will be affected by nanomedicine are regulatory agencies and healthcare delivery systems. 
The responsibility of the FDA is to protect the health of the public by ensuring that biological products, cosmetics, national food supplies, medical supplies, and drugs are safe. ${ }^{24}$ Currently, the FDA has not developed specific regulations for products containing nanomaterials; however, many products containing engineered nanomaterials do fall under their jurisdiction. These range from sunscreens to prescription drugs, which are raising concern among the public and lawmakers.

In 2006, a legal petition was filed by the International Center for Technology Assessment and other concerned consumers groups against the FDA for a perceived "lack of initiative" in regulating nanomaterial-containing products under their jurisdiction. ${ }^{25}$ In response to the legal petition, the FDA created a "Nano Task Force". The task force produced a document entitled Nanotechnology: A Report of the U.S. Food and Drug Administration Task Force detailing how the FDA is addressing products containing engineered nanosized particles. ${ }^{26}$ Ultimately, the document states that they do not have sufficient health and safety information to treat nanosized particles differently from larger particles of the same material composition. In the above report, the FDA recommends that labeling of nanoparticle-containing products should be evaluated on a case-by-case basis. However, they do not believe that the current state of the science proves that nanosized particles are cause for safety concern, and therefore do not need to be specifically labeled as such. ${ }^{26}$ Despite concern from advocacy groups like the Consumers Union, there have been no changes to their method of review for products containing nanomaterials. Additional and appropriate toxicological data must be generated to effectively inform the regulation of products entering the market. A balance between protecting consumers and fostering innovation is necessary to responsibly advance nanotechnology.

The challenge of regulating engineered nanomaterials is not limited to the FDA. Other United States federal agencies that are also under pressure to ensure the safe development of nanotechnology include the Environmental Protection Agency (EPA) and the Occupational Safety and Health Administration. One significant challenge these agencies face is the lack of standardization of nanomaterials. The diverse nature of nanomaterials does not lend itself well to the current nomenclature used for chemicals. Material safety datasheets, which play a critical role in worker safety, are one example of how a standardized naming system is used to communicate health and safety information. From a policy standpoint, it is critical to have a universal and consistent naming system to begin developing regulations pertaining to particular engineered nanomaterials. Another current challenge for these federal entities is that knowledge gaps exist regarding the potential environmental and human health effects related to exposure to engineered nanomaterials. Health and safety research is lagging behind the rapid development of new nanotechnologybased products. Limited federal funding to agencies charged with this type of research, such as the National Institute for Occupational Safety and Health (NIOSH), FDA, and EPA, is contributing to this lag. In 2009, NIOSH received approximately $0.4 \%$ of the total National Nanotechnology Initiative's (NNI's) budget. Their 2011 funding request is about two and a half times the 2009 budget and would comprise almost $1 \%$ of the total 2011 NNI budget. ${ }^{27}$ Other regulatory agencies are also requesting increased funding; this federal financial support is critical to fill the health and safety knowledge gaps and inform policymakers.

Advances in nanomedicine will also affect healthcare delivery systems. Screening modalities with higher sensitivity and specificity for disease detection could greatly improve prognoses and decrease healthcare costs. Initially, there could be resistance from insurance providers to pay for certain treatments using nanomedicine. New technologies are often more expensive than traditional medical interventions, and coverage is often opposed even though the new methods may be more effective. This is a challenge that will be overcome with time and sufficient data to clearly demonstrate the benefits of new nanomedicine applications in the prevention, diagnosis, and treatment of disease. Healthcare providers and delivery systems could face financial challenges as they will bear the initial burden of purchasing new equipment and dedicating their time to learn about advances in nanomedicine in order to incorporate them into clinical practice. These real as well as perceived barriers should be overcome through a collaborative effort from all involved stakeholders. Public health professionals will play a key role in working with researchers who develop the technology to transparently disseminate information regarding risks, costs, and benefits to those who will implement them.

\section{Social and community behavior}

Often individuals' health decisions are influenced by their perception of risks and benefits despite factual information. The health belief model describes this theory, and more specifically, how the media plays a role in decision making. ${ }^{28}$ With books like Engines of Creation by Eric Drexler and Prey by Michael Crichton proposing that nanorobots will eventually take over the world, nanotechnology has been given a negative spin in the eyes of some. ${ }^{29,30}$ Current studies 
suggest that there is skepticism among the public about the safety of nanotechnology. ${ }^{31}$ In particular, nanomedicine may fall under more scrutiny than nanotechnology in general because its applications often take the form of direct medical interventions. Over the course of history, there are examples of emerging technologies that have been greatly hindered or even failed due to negative public and social perception. For example, genetically modified foods are considered to be safe for consumption, but due to negative public perception, the industry was not able to survive. ${ }^{32,33}$ Noncompliance with vaccinations due to public fear of adverse health effects is another example of how perception can affect medical decision making as well as the health of entire populations. ${ }^{34}$ Stakeholders in nanomedicine must be proactive with regard to clear, consistent, and transparent communication with the lay public and policymakers. Strategic risk communication with the public is essential, especially in light of pre-existing stigma and fear about nanotechnology.

\section{Environmental health}

Understanding the environmental and human health implications of engineered nanomaterials, products, and byproducts and the processes involved in making them are critical to proactively explore. Early stage research implies that certain nanoparticles may be more persistent in the environment than others, highlighting the need to collect more information regarding risk assessment and management. ${ }^{35}$ Environmental regulation of nanomaterials falls under the EPA's Toxic Substance Control Act. ${ }^{36}$ While there are over 1000 nanoenabled consumer products already on the market, knowledge gaps exist regarding their fate and transport within humans, the environment, and ecosystems. ${ }^{37}$ For example, many topical creams such as sunscreens that are already on the market contain titanium dioxide nanoparticles. ${ }^{38}$ Sunscreen brands that contain nanoparticles include Burt's Bees ${ }^{\circledR}$, Banana Boat $^{\circledR}$, Coppertone ${ }^{\circledR}$, and many others. ${ }^{39}$ These products are intended for direct application to the skin where some of the material will be absorbed through the epidermis and some will be washed or rubbed off and enter public wastewater systems. Another particle of concern is nanosilver due to its inherent antimicrobial nature and potential to alter the wastewater treatment environment. Water treatment facilities have not been specifically designed for removal of nanoparticles. ${ }^{40}$ Analysis of the entire life-cycle of engineered nanomaterials, from their raw materials to disposal, is an important part of responsibly advancing nanotechnology from a health and safety perspective. ${ }^{41}$ As the number of nanomaterial-containing products on the market continues to rise, there is increased awareness and concern regarding their environmental impact.

Similarly, nanoparticles used for medical interventions will have multiple points of entry into the environment based on their particular application, function, and disposal. This may include excreted nanoenabled drug products into wastewater, disposal of novel imaging agents, and medical devices at their end of life in a landfill. Currently, the FDA and EPA provide guidelines for the public on how to dispose of unused medications, as certain drugs have the potential to alter the wastewater treatment system. ${ }^{42,43}$ Therefore, it is critical to understand how nanopharmaceuticals will affect water treatment as well. Continued research is necessary to understand the impact of nanomaterials on the environment throughout all stages of their life-cycle.

\section{Training}

Public health professionals, medical practitioners, and scientific researchers involved with developing and deploying nanomedicine applications should be versed in the basic concepts and principles of public health as they relate to emerging technologies. This will drive research in a direction that will most efficiently impact population health and allow for effective communication and collaboration between stakeholders from industry, government, and academia. The cohort charged with advancing nanomedicine will comprise many different types of professionals, which will necessitate truly interdisciplinary collaboration.

To assist with the implementation of emerging nanomedicine applications into healthcare systems, public health workers will need to be familiar with the concepts of nanotechnology. Public health professionals should be educated about the growing field of nanomedicine and its implications for population health. This can occur through multiple mechanisms such as increasing the literature base through publication of nanomedicine articles directed towards the public health audience, incorporating sessions into major conferences such as the American Public Health Association annual conference, and offering seminars and workshops solely dedicated to educating public health professionals about the advances in nanomedicine. Subsequently, those involved with strategic planning of the future of nanomedicine within government, industry, and academia should understand its potential to impact public health.

Education for clinicians will also be essential to effectively deploy these nanoenabled medical technologies in clinical practice. Applications in certain medical specialties and subspecialties such as radiology, infectious disease, and oncology 
are currently racing ahead from research and development to commercial availability. The need for additional training and education in these medical specialty areas is more urgently needed than for other fields at this time; however, it is likely that all medical specialties will be directly impacted in the coming years. Expertise for developing educational and training modules for medical providers will need to come from a group that can understand and synthesize information from basic research and clinical medicine.

Training and education amongst all groups will need to occur in a cyclic manner. Public health professionals must become educated about nanotechnology and its advances in medicine to, in turn, educate researchers and medical professionals about its national and international impact on population health. This will require the formation of partnerships across disciplines and sectors resulting in more efficient translation of technology to patient care, which will ultimately improve individual and population health outcomes.

In addition, a new generation of physician scientists who are literate in both nanotechnology and medicine will propel new nanomedicine innovations into practice. As the art and science of medicine evolves rapidly in the face of cuttingedge technologies, a new generation of clinical researchers is needed to integrate and accelerate the potential impact of these advances on human health. This truly hybrid cohort will play an integral role in driving clinical translational nanomedicine research from bench top to bedside. According to the National Nanotechnology Initiative, a solid educational foundation, a skilled workforce, and a state-of-the-art research and development infrastructure are essential in this regard. ${ }^{44}$ Nanoscale science, engineering, and technology programs and resources are required to produce a new generation of researchers and inventors working at the nanoscale. Specific efforts should include the development of educational modules that incorporate nanotechnology into curricula for many disciplines contributing to nanotechnology. ${ }^{44}$ Many national groups and strategic federal initiatives, including the National Cancer Institute Alliance for Nanotechnology, specifically call for training programs designed to produce the next generation of nanomedicine researchers and encourage young people to pursue cross-disciplinary careers in science, medicine, and engineering. ${ }^{45}$

\section{Conclusion}

The opportunities for nanomedicine to improve health are limitless. To maximize gains in individual and population health, inclusion of public health expertise is essential.
This influence in the development of nanomedicine will help to identify the greatest areas of need for technological innovation, determine how to best allocate funding, and shape polices to protect humans and the environment.

An important part of advancing nanomedicine is expanding cross-disciplinary training for researchers, medical care providers, and public health professionals working in industry, government, and academia. Taking a collaborative approach to nanomedicine research and education will advance the state of the science efficiently, resulting in a greater return on investment for public health. Research and development of novel nanomedicine applications integrated with public health principles will transform human health across the world.

\section{Disclosure}

The authors report no conflicts of interest in this work.

\section{References}

1. Aschengrau A, Seage G. Essentials of Epidemiology in Public Health. Sudbury, MA: Jones and Bartlett Publishers, Inc; 2008.

2. National Nanotechnology Initiative. What is Nanotechnology? Available from: http://www.nano.gov/html/facts/whatIsNano.html. Accessed 2010 Apr 6.

3. Bawarski WE, Chidlowsky E, Bharali DJ, Mousa SA. Emerging nanopharmaceuticals. Nanomedicine. 2008;4:273-282.

4. Kostarelos K, Bianco A, Prato M, et al. Promises, facts and challenges for carbon nanotubes in imaging and therapeutics. Nat Nanotechnol. 2009;4(10):627-633.

5. Oberdorster G, Maynard A, Donaldson K, et al. Principles for characterizing the potential human health effects from exposure to nanomaterials: elements of a screening strategy. Part Fibre Toxicol. 2005;2(8):1-35.

6. Winslow CEA. The untilled field of public health. Modern Medicine. 1920;2:183-191.

7. Association of Schools of Public Health (ASPH). MPH Core Competency Model, 2006. Available from: http://www.asph.org/document. $\mathrm{cfm}$ ?page $=851$. Accessed 2010 Apr 3 .

8. Friis R. Essentials of Environmental Health. Sudbury, MA: Jones and Bartlett Publishers, Inc; 2007.

9. World Health Organization. Hepatitis B [updated 2010]. Available from: http://www.who.int/mediacentre/factsheets/fs204/en/. Accessed 2010 Jun 18.

10. Feng L, Qi X, Zhou X, et al. Pharmaceutical and immunological evaluation of a single-dose hepatitis $\mathrm{B}$ vaccination using PLGA microspheres. $J$ Control Release. 2006;112:35-42.

11. American Cancer Society. Cancer Statistics 2009 Presentation [updated 2010]. Available from: http://www.cancer.org/docroot/pro/ content/pro_1_1_cancer_statistics_2009_presentation.asp. Accessed 2010 Apr 6.

12. National Cancer Institute. A Snapshot of Nanotechnology [updated 2009 Sep]. Available from: http://www.cancer.gov/aboutnci/servingpeople/ snapshots/nanotech.pdf. Accessed 2010 Jul 7.

13. National Cancer Institute Alliance for Nanotechnology in Cancer. Learn About Nanotechnology in Cancer. Available from: http://nano.cancer. gov/about/mission/faq.asp. Accessed 2010 Apr 6.

14. The Project on Emerging Nanotechnologies. Cancer [updated 2010]. Available from: http://www.nanotechproject.org/inventories/medicine/ apps/cancer/. Accessed 2010 Apr 6.

15. Ferrari M. Cancer nanotechnology: opportunities and challenges. Nature Reviews Cancer. 2005;5:161-171. 
16. Center for Disease Control and Prevention. FASTSTATS - Leading Causes of Death [updated 2009 Dec 31]. Available from: http://www. cdc.gov/nchs/fastats/deaths.htm. Accessed 2010 Jul 28.

17. Engel E, Michiardi A, Navarro M, Lacroix D, Planell J. Nanotechnology in regenerative medicine: the materials side. Trends Biotechnol. 2008; 26:39-47.

18. Tran P, Zhang L, Webster T. Carbon nanofibers and carbon nanotubes in regenerative medicine. Adv Drug Deliv Rev. 2009;61(12):1097-1114

19. Oliveria J, Salgado A, Mano J, Reis R. Dedrimers and derivatives as a potential therapeutic tool in regenerative medicine - a review. Prog Polym Sci. In press 2010.

20. Rosner B. Fundamentals of Biostatistics. 6th ed. Belmont (CA): Thomson Higher Education; 2006

21. Bawarsk W, Childowsky E, Bharali D. Emerging nanopharmaceuticals. Pharmacology. 2008;4:273-282.

22. Sakamoto J, van de Ven A, Godin B, et al. Enabling individualized therapy through nanotechnology. Pharmacol Res. 2010;62(2):57-89.

23. Normura S, Kondo M, Nagano M, Matsui K, Egashira T. Development of a novel nano-Invader DNA chip system. J Biochem Biophys Methods. 2007;5(1):787-795.

24. US Food and Drug Administration (FDA). Centers and Offices, 2010. Available from: http://www.fda.gov/AboutFDA/CentersOffices/default. htm. Accessed 2010 Apr 5.

25. Kimbrell GA. CTA International Center for Technology Assessment FDA Public Meeting on Nanotechnology [updated 2006 Oct 10]. Available from: http:/www.fda.gov/ohrms/dockets/dockets/06n0107/06n0107-ts00008-Kimbrell.ppt. Accessed 2010 Jul 27.

26. US Food and Drug Administration (FDA). Nanotechnology: a report of the U.S. Food and Drug Administration Nanotechnology Task Force [updated 2007]. Available from: http://www.fda.gov/downloads/ ScienceResearch/SpecialTopics/Nanotechnology/ucm110856.pdf. Accessed 2010 Apr 5.

27. National Nanotechnology Initiative. Funding. Available from: http:// www.nano.gov/html/about/funding.html. Accessed 2010 Jun 18.

28. Encyclopedia of Public Health. Health Belief Model [update 2010]. Available from: http://www.enotes.com/public-health-encyclopedia/ health-belief-model. Accessed 2010 Jun 18.

29. Drexler KE. Engines of Creation: The Coming Era of Nanotechnology. New York: Anchor Books; 1986

30. Crichton M. Prey. New York (NY): Harper Collins; 2002.

31. Satterfield T, Kandlikar M, Beaudrie C, et al. Anticipating the perceived risk of nanotechnologies. Nat Nanotechnol. 2009;4:752-759.

32. Frewer L, Lassen J, Kettliz B, et al. Societal aspects of genetically modified foods. Food Chem Toxicol. 2004;42:1181-1193.
33. Gaskell G, Allum N, Wagner W, et al. GM foods and the misperception of risk perception. Risk Anal. 2004;1:185-194.

34. May T. Public communication, risk perception, and the viability of preventive vaccination again communicable diseases. Bioethics. 2005; 19(4):407-421.

35. Kahru A, Dubourguier HC. From ecotoxicology to nanoecotoxicology. Toxicology. 2010;269:105-119.

36. U.S. Environmental Protection Agency: Pollution Prevention and Toxics. Control of Nanoscale Materials under the Toxic Substance Control Act [updated 2010 Apr 22]. Available from: http://www.epa. gov/oppt/nano/. Accessed 2010 Jun 18.

37. The Project on Emerging Nanotechnologies. Consumer Products [updated 2010]. Available from: http://www.nanotechproject.org/ inventories/consumer/. Accessed 2010 Apr 5.

38. Hallock F, Greenley P, DiBerardinis L, Kallin D. Potential risks of nanomaterials and how to safely handle materials of uncertain toxicity. J Chemical Health Safety. 2009 Jan/Feb:16-23.

39. The Project on Emerging Nanotechnologies. Health and fitness [updated 2010]. Available from: http://www.nanotechproject.org/inventories/ consumer/browse/categories/health_fitness/sunscreen/page3/.Accessed 2010 Apr 5.

40. Bystrzejewska-Piotrowska G, Golimowski J, Urban P. Nanoparticles: their potential toxicity, waste and environmental management. Waste Manag. 2009;29:2587-2595.

41. Som C, Berges M, Chaudhry Q, et al. The importance of lice cycle concepts for the development of safe nanoproducts. Toxicology. In press 2010.

42. U.S. Food and Drug Administration. Disposal by Flushing of Certain Unused Medicines: What You Should Know. U.S. Department of Human and Health Services [updated 2010 Apr 4]. Available from: http://www. fda.gov/drugs/resourcesforyou/consumers/buyingusingmedicinesafely/ ensuringsafeuseofmedicine/safedisposalofmedicines/ucm186187.htm. Accessed 2010 Jun 18.

43. U.S. Environmental Protection Agency: Pharmaceuticals and Personal Care Products Drug Disposal and Stewardship. Ramifications for the Environment and Human Health [updated 2010 May 25]. Available from: http://www.epa.gov/ppcp/projects/disposal.html. Accessed 2010 Jun 18.

44. National Nanotechnology Initiative. Strategic Plan [updated 2007]. Available from: http://www.nano.gov/html/about/strategicplan.html. Accessed 2010 Apr 5.

45. NCI Alliance for Nanotechnology in Cancer. Training [updated 2010]. Available at: http://nano.cancer.gov/collaborate/training/. Accessed 2010 Apr 5.
International Journal of Nanomedicine

\section{Publish your work in this journal}

The International Journal of Nanomedicine is an international, peerreviewed journal focusing on the application of nanotechnology in diagnostics, therapeutics, and drug delivery systems throughout the biomedical field. This journal is indexed on PubMed Central,

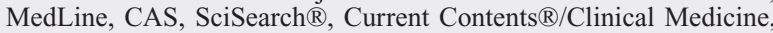

\section{Dovepress}

Journal Citation Reports/Science Edition, EMBase, Scopus and the Elsevier Bibliographic databases. The manuscript management system is completely online and includes a very quick and fair peer-review system, which is all easy to use. Visit http://www.dovepress.com/ testimonials.php to read real quotes from published authors. 